\title{
Safety argumentation for automated driving systems
}

Michael Helmle, P. Sautter, F. Hauler, F. von Zeppelin, Robert Bosch GmbH

This manuskript is not available according to publishing restriction.

Thank you for your understanding.

(C) Springer Fachmedien Wiesbaden 2016, M. Bargende, H.-C. Reuss, J. Wiedemann (Hrsg.), 16. Internationales Stuttgarter Symposium, Proceedings, DOI 10.1007/978-3-658-13255-2_105 